\title{
Aspectos Genéticos e Ambientais da Curva de Lactação de Vacas da Raça Guzerá1
}

\author{
Jaime Araujo Cobuci ${ }^{2}$, Ricardo Frederico Euclydes ${ }^{3}$, Roberto Luiz Teodoro 4 , \\ Rui da Silva Verneque ${ }^{4}$, Paulo Sávio Lopes ${ }^{3}$, Martinho de Almeida e Silva ${ }^{5}$
}

\begin{abstract}
RESUMO - O conhecimento das relações genéticas entre os parâmetros da curva de lactação e a produção de leite é importante para a seleção de vacas e de touros. Os registros de produção de leite de 583 vacas da raça Guzerá, filhas de 165 reprodutores, foram usados para estimação dos parâmetros da curva de lactação em dois modelos matemáticos. As informações referentes às produções de leite foram obtidas usando-se a base de dados extraída do Arquivo Zootécnico Nacional, mantido na Empresa Brasileira de Pesquisa Agropecuária (Embrapa Gado de Leite). As análises foram efetuadas usando-se o sistema MTDFREML, sob modelo animal que incluiu os efeitos fixos de rebanho-ano-estação de parto e, como covariável, a idade da vaca ao parto, com termos linear e quadrático. Como efeitos aleatórios foram incluídos animal, efeito permanente de ambiente e erro. As estimativas de correlações genéticas entre a produção de leite total, a produção inicial e a taxa de declínio da produção foram de 0,55 e 0,86 e de - 0,19 e 0,38 , respectivamente, para cada modelo matemático estudado. Para as duas últimas características, estas estimativas foram de 0,45 e 0,75 , indicando que vacas com maiores produções iniciais apresentaram declínios mais acentuados da produção de leite ao longo do período de lactação. As correlações genéticas entre a produção de leite em 305 dias e os parâmetros do modelo seguiram a mesma tendência, em magnitude e sinal, às da produção de leite total. De acordo com os resultados, pode-se inferir que a seleção para a produção de leite total resulta em acréscimo na produção inicial e mudanças inexpressivas na taxa de declínio da produção de leite, não alterando a forma da curva de lactação. As correlações de ordem entre as classificações dos valores genéticos preditos foram altas e positivas $(0,68$ e 0,74$)$, sugerindo que os indivíduos com os maiores valores genéticos para produção inicial de leite seriam também aqueles com maiores valores para produção de leite total.
\end{abstract}

Palavras-chave: correlações genéticas, curva de lactação, método REML, modelos matemáticos, raça Guzerá

\section{Genetic and Environmental Aspects of the Lactation Curve in Guzerá Breed Cows}

ABSTRACT - The knowledge of the genetic relationships between the parameters of lactation curve and the milk yield is important for the selection of cows and sire. The records milk yield from 583 cows of the Guzerá breed, daughters of 165 sire, were used to estimate the lactation curve parameters by two mathematical models. Milk productions were obtained from the extracted base of data from National Animal Science Archive maintained by National Center for Dairy Research (Embrapa Gado de Leite). The analyses were performed by the MTDFREML system, under animal model containing herd-year-season of calving, the linear and quadratic age effects of calving, as fixed effects animal, permanent environment and error included as random effects. The estimates of genetic correlations between the total milk yield and the initial milk yield, and the rate of decline of milk yield were 0.55 and 0.86 , and -0.19 and 0.38 , respectively, for each mathematical model. For last two characteristics, these estimates were 0.45 and 0.75 , indicating that cows with larger initial milk yield presented accentuated milk yield declines along the lactation period. The genetic correlations among 305-days yield and the parameters of model followed the same tendency, in magnitude and sign, to the one of the total milk yield. In agreement with the results, it can be inferred that the selection for the total milk yield results in increment in the initial milk yield, and in inexpressive changes in the rate of decline of the milk yield, not altering the form of the lactation curve. The rank correlations among the classifications of the predicted genetic values were high and positive ( 0.68 and 0.74 ), suggesting that the individuals with the highest genetic values for initial milk yield, would be also those with higher values for total milk yield.

Key Words: genetic correlations, Guzera breed, lactation curve, mathematical model, REML

\section{Introdução}

A curva de lactação pode ser definida como a representação gráfica da produção de leite de uma vaca no decorrer de sua lactação. O estudo sobre curvas de lactação pode contribuir para o melhor entendimento do sistema de produção, pois o conhecimento da forma da curva e suas implicações sobre a produção de leite pode auxiliar o produtor na previsão da produção de leite de suas vacas em

\footnotetext{
${ }^{1}$ Parte da Dissertação apresentada à UFV, pelo primeiro autor, para obtenção do grau de "Magister Scientiae" em Zootecnia

2Zootecnista, Estudante de Doutorado da UFV, Bolsista da CAPES. E.mail: ds35625@correio.cpd.ufv.br

3 Professores da Universidade Federal de Viçosa.

${ }^{4}$ Pesquisador do CNPGL/EMBRAPA, Juiz de Fora, MG.

5 Professor da Universidade Federal de Minas Gerais.
} 
determinado estádio de lactação e, também, na tomada de decisões quanto ao descarte ou manejo.

Este tipo de estudo teve início em 1923, quando Brody propôs o primeiro modelo matemático com o objetivo de descrever a curva de lactação. Desde então, inúmeros trabalhos foram publicados, sendo que poucos estudaram os fatores genéticos relacionados com os parâmetros dos modelos.

Nesse sentido, o conhecimento das correlações entre os parâmetros da curva de lactação é importante ao se formularem programas de seleção. Por isto, torna-se necessário conhecer a magnitude e a direção (positiva ou negativa) das correlações entre as características de interesse, pois a seleção para uma característica poderá provocar mudanças em outra.

No Brasil, CARDOSO et al. (1986), analisando dados de produção de leite de vacas da raça Gir, obtiveram o valor 0,81 para correlação genética entre a produção inicial e a produção de leite total. No entanto, BIANCHINI SOBRINHO e DUARTE (1988), para a mesma raça, obtiveram valores de 0,34 e 0,51 , respectivamente, para as correlações genéticas entre os parâmetros " $a$ " e " $c$ " e a produção de leite total de vacas de primeira e segunda lactações.

Utilizando métodos bayesianos, VARONA et al. (1998) obtiveram correlações genéticas de $0,71,0,16 \mathrm{e}$ $-0,04$, respectivamente, para os parâmetros " $a$ ", “ $b$ " e " $c$ " do modelo Gama e a produção de leite total.

Em animais de raças européias, SCHNEEBERGER (1981) e FERRIS et al. (1985) estimaram valores para a correlação genética entre a produção de leite em 305 dias e os parâmetros de diferentes modelos, que variaram de $-0,37$ a 0,37 para o parâmetro " $a$ ", de 0,02 a 0,40 para o " $b$ " e de 0,00 a $-0,03$ para o parâmetro " $c$ ".

Vários trabalhos têm relatado a influência dos fatores de ambiente sobre a forma da curva de lactação, dentre os quais ano e estação de parto, idade da vaca ao parto, ordem de parto, período de serviço e duração da lactação são citados como os que mais afetam a produção inicial, a taxa de declínio da produção e a produção de leite total (GROSSMAN et al., 1986; DURÃES et al., 1991; LOPES et al., 1996; e JUNQUEIRA et al., 1997).

O objetivo do presente estudo foi analisar a forma da curva de lactação e suas implicações sobre a produção de leite de vacas da raça Guzerá, além de avaliar as diferenças nas estimativas dos parâmetros da curva de lactação obtidas por dois modelos matemáticos.

\section{Material e Métodos}

Os dados de produção de leite utilizados neste estudo foram obtidos de nove rebanhos Guzerá, localizados nas Regiões Nordeste e Sudeste do Brasil, totalizando 1130 lactações com controles mensais, de 583 vacas, referentes ao período de 1983 a 1997. Esses dados fazem parte do Arquivo Zootécnico Nacional, mantido na Embrapa Gado de Leite, Juiz de Fora - MG, e foram gerados em parceria com a Associação Brasileira dos Criadores de Zebu (ABCZ).

Os modelos utilizados no ajuste às produções individuais de leite de vacas Guzerá foram:

$$
\begin{aligned}
& y=a n e^{-c n} \\
& y=a-c n+\ln (n)
\end{aligned}
$$

O modelo (1), descrito por PAPAJCSIK e BODERO (1988), é uma versão do modelo Gama de Wood (1967), pois considera o parâmetro "b", do modelo original, igual a um. O modelo (2) foi descrito por COBUCI et al. (2000). Considere para ambos os modelos a variável " $y$ " como a produção de leite no estádio " $n$ " da lactação, em que " $n$ " é o período de tempo decorrido do parto até a data em que foi medida a produção de leite (em meses); " $a$ ”, a produção inicial de leite; e " $c$ ”, a taxa de declínio da produção de leite durante o período da lactação.

A estimação dos parâmetros de cada modelo foi realizada por meio de regressão não-linear da produção de leite, em função do número de dias do parto até a avaliação do controle leiteiro (em meses), utilizando-se o método de Gauss Newton.

Na estimação dos componentes de (co)variância necessários para a estimação dos parâmetros genéticos das características, foram utilizados o método da Máxima Verossimilhança Restrita (REML) e o modelo animal, por intermédio do sistema MTDFREML (Multiple Trait Derivate Free Restricted Maximum Likelihood), descrito por BOLDMAN et al. (1995). A estratégia de utilização empregada foi semelhante à de VERNEQUE (1994), em que as análises com uma só característica são rodadas, inicialmente, para obtenção de boa aproximação dos componentes de variância. Os componentes de variância e covariância obtidos são utilizados como informação inicial na estimação conjunta com baixa precisão $\left(\operatorname{Var}\left(-2 \log \left(\mathrm{L}_{2}\right)\right)<10^{-6}\right)$. As estimativas obtidas são então utilizadas, sucessivamente, como informação inicial de processamentos mais precisos (Var $\left.\left(-2 \log \left(\mathrm{L}_{2}\right)\right)<10^{-9}\right)$. Após o estabelecimento deste procedimento, para evitar a ocorrência de um má- 
1206 Rev. bras. zootec.

ximo local, foram realizados novos processamentos, até que não se verificassem mais variações superiores a 0,002 unidades nos valores da função de verossimilhança, entre dois processamentos sucessivos.

Em análises com duas características, o modelo linear misto utilizado em sua forma matricial foi:

$$
\mathrm{y}=\mathrm{X} \beta+\mathrm{Z}_{\mathrm{g}}+\mathrm{W}_{\mathrm{p}}+\mathrm{e}
$$

em que y é o vetor das variáveis dependentes (produção de leite total, produção inicial e taxa de declínio da produção); $X$, a matriz de incidência de efeitos fixos; $\beta$, o vetor de efeitos fixos (rebanho-ano-estação de parto, idade da vaca ao parto com termos linear e quadrático); $\mathrm{Z}$, a matriz de incidência dos efeitos genéticos diretos; g, o vetor dos efeitos aleatórios dos valores genéticos diretos do animal; $\mathrm{W}$, a matriz de incidência dos efeitos permanentes de ambiente; $p$, o vetor dos efeitos permanentes de ambiente; $\mathrm{e}, \mathrm{o}$ vetor dos erros aleatórios associados a cada observação.

As produções de leite totais estimadas pelos modelos de regressão (PLTE) foram obtidas por:

$$
\text { PLTE }=\left(\sum_{n=1}^{t} \hat{y}_{n}\right) / t \text {. Plact }
$$

em que $\hat{y}_{n}=$ produção mensal de leite estimada $(n=1,2, \ldots, \mathrm{t}) ; n=$ número de controles (em meses); e Plact $=$ período de lactação (em dias).

\section{Resultados e Discussão}

As estimativas dos coeficientes de determinação, o percentual de curvas atípicas, percentual de parâmetros significativos do modelo matemático, raiz do quadrado médio do resíduo da regressão e os desvios médios, em porcentagem (\%) e quilos de leite (kg), obtidos com a utilização das lactações individuais, estão apresentados na Tabela 1. Constam da Tabela 2 os modelos, as produções de leite totais estimadas e os desvios, obtidos por meio da curva de lactação média. Com base nos desvios, pode-se observar que o modelo 2 estimou produções bem próximas às das produções observadas.

Os resíduos estimados como a diferença entre as produções de leite observadas e as estimadas pelos modelos ora subestimaram ora superestimaram as produções em diversos estádios da curva de lactação, indicando a presença de correlação serial positiva entre os sucessivos resíduos. Entretanto, para o modelo 2, estes resíduos foram muito reduzidos nos primeiros oito estádios de lactação (Figura 1).

Esta forma de correlação pode ocorrer em curvas de lactação, porque as observações estão ordenadas em função do tempo e, de certa forma, alguns autores consideram normal a presença de correlação residual, pois os resíduos contêm toda a variação não-explicada pelo modelo, relativas aos efeitos genéticos e de ambiente permanente (EL FARO et al., 1999).

Na Tabela 3 são apresentadas as distribuições dos coeficientes de determinação (em porcentagem), de acordo com os modelos propostos e a ordem de parto das vacas. Verifica-se que os modelos apresentaram piores ajustes às produções de leite das vacas de primeira ordem de parto, sendo que somente 19,1 e $46,9 \%$ dos coeficientes de determinação obtidos pelos modelos 1 e 2 , respectivamente, foram superiores a 0,8 . Resultado semelhante foi relatado por KELLOGG et al. (1977), em trabalho com dados de vacas da raça Holandesa. Segundo os autores, os piores ajustes obtidos para as vacas de primeira lactação poderiam ser explicados pelo achatamento da curva, sem que evidenciasse o pico de produção nos primeiros meses de lactação.

Tabela 1 - Coeficiente de determinação $\left(R^{2}\right)$, percentual de curvas atípicas (\%At), percentual de parâmetros significativos (\%Sign), número de observações $(\mathrm{N})$, desvios (\% e kg) e raiz do quadrado médio do resíduo $(\sqrt{Q M R})$, obtidas pelos diferentes modelos

Table 1 - Coefficient of determination $\left(R^{2}\right)$, percentage of atypical curves (\%At), percentage of significant parameters (\%Sign), number of observations $(\mathrm{N})$, deviations $(\%$ and $\mathrm{kg}$ ) and root of the least square of the residue $\sqrt{Q M R}$, obtained by the different models

\begin{tabular}{lccccccc}
\hline $\begin{array}{l}\text { Modelo } \\
\text { Model }\end{array}$ & $\mathrm{R}^{2}$ & \% At & \% Sign & $\mathrm{N}$ & $\begin{array}{c}\text { Desvio }(\%)^{1} \\
\text { Deviation }(\%)\end{array}$ & $\begin{array}{c}\text { Desvio }(\mathrm{kg}) \\
\text { Deviation }(\mathrm{kg})\end{array}$ \\
\hline 1 & 0,56 & 0,00 & 99,68 & 1130 & 4,14 & $1, \sqrt{Q M R}$ \\
2 & 0,72 & 0,62 & 97,60 & 1123 & 0,79 & 15,81 & 1,15 \\
\hline
\end{tabular}

1 Desvio \%=[(PLTO-PLTE)/PLTO]x100 (Deviation \% = [(PLTO-PLTE)/PLTO]x100).

PLTO = Produção de leite total; PLTE = Produção de leite total estimada (PLTO = Total milk yield; PLTE = Estimated total milk yield). 
Tabela 2 - Modelo, produção de leite total estimada (PLTE) e desvios da produção de leite (\% e kg) das vacas Guzerá, de acordo com os modelos

Table 2 - Model, total milk yield (PLTE) and milk yield deviations (\% and $\mathrm{kg}$ ) of Guzera cows, according to the models

\begin{tabular}{lccc}
\hline $\begin{array}{l}\text { Modelo } \\
\text { Model }\end{array}$ & PLTE & $\begin{array}{c}\text { Desvios } \\
(\%)^{1} \\
\text { Deviation } \\
(\%)^{1}\end{array}$ & $\begin{array}{c}\text { Desvios } \\
(\mathrm{kg}) \\
\text { Deviation } \\
(\mathrm{kg})\end{array}$ \\
\hline$\hat{y}=10,996 . \mathrm{n} \cdot \mathrm{e}-0,356 . \mathrm{n}$ & 2258,9 & 4,25 & 100,50 \\
$\hat{y}=11,383-0,938 \cdot \mathrm{n}+1 \mathrm{n} .(\mathrm{n})$ & 2343,6 & 0,67 & 15,79 \\
\hline
\end{tabular}

${ }^{1}$ Desvio \% $=[(2.359,4-\mathrm{PLTE}) / 2359,4] \times 100$.

Deviation \% $=[(2359.4-P L T E) / 2,359.4] \times 100$.

Na Figura 2, estão representadas as curvas de lactação estimadas pelos modelos matemáticos propostos e a curva média observada, assim como a curva de lactação atípica estimada pelo modelo 2. A produção média diária observada foi de $8,11 \mathrm{~kg}$, com produção inicial de $10,58 \mathrm{~kg}$ de leite e período médio de lactação de 290 dias. Formas atípicas de curva de lactação são decorrentes das estimativas negativas para os parâmetros do modelo. Neste caso, este formato foi devido às estimativas negativas obtidas para o parâmetro " $c$ ".

A produção de leite total observada, o período de lactação e as estimativas dos parâmetros " $a$ " e " $c$ ", segundo a ordem de parto, estão apresentados na Tabela 4. Observa-se que as vacas mais jovens tiveram menores produções inicial e maiores persistências da lactação.

Estes resultados estão de acordo com JUNQUEIRA et al. (1997) e QUEIROZ et al. (1991),

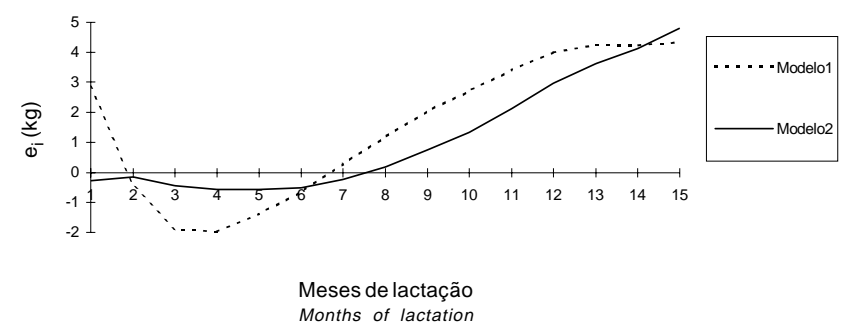

Figura 1 - Gráfico de dispersão dos resíduos estimados $\left(\mathrm{e}_{\mathrm{j}}\right)$ para a curva de lactação média das vacas Guzerá, de acordo com os modelos.

Figure 1 - Dispersion graphic of estimated $\left(e_{j}\right)$ errors for the average lactation curve, according to the model, for Guzera cows. segundo os quais vacas mais jovens apresentaram menores níveis de produção ao longo do período de lactação, com decréscimos menores que garantem uma persistência mais elevada. Contudo, com o avançar da idade e, conseqüentemente, com a maturidade fisiológica dos animais, melhores desempenhos são alcançados.

$\mathrm{Na}$ Tabela 5 estão apresentadas as produções de leite total observadas e as estimativas do parâmetro " $c$ ", de acordo com a classe de produção inicial. Vacas que produziram menores quantidade de leite no início da lactação apresentaram lactações com declínios menos acentuados da produção diária de leite. No entanto, as vacas pertencentes às classes de maiores produções iniciais apresentaram maiores taxas de declínio da produção e maiores produções na lactação.

Na Tabela 6 são apresentadas as produções de leite total observadas e as estimativas obtidas para os parâmetros dos modelos, segundo a classe de período de lactação das vacas. Observa-se que os modelos apresentaram tendências diferentes quanto a produção inicial nas diversas classes de período de lactação. Para o modelo 1 , as estimativas de produção inicial tenderam a diminuir com o aumento no período de lactação, o mesmo não aconteceu com o segundo modelo. Portanto, maiores produções de leite parecem estar associadas a menores taxas de declínio da produção.

JUNQUEIRA et al. (1997), na raça Holandesa, verificaram que animais com duração da lactação entre 210 e 244 dias apresentaram menores produções iniciais e maiores declínios da produção, quando comparadas a animais de classes de duração de lactação maiores, que normalmente apresentam maiores persistências.

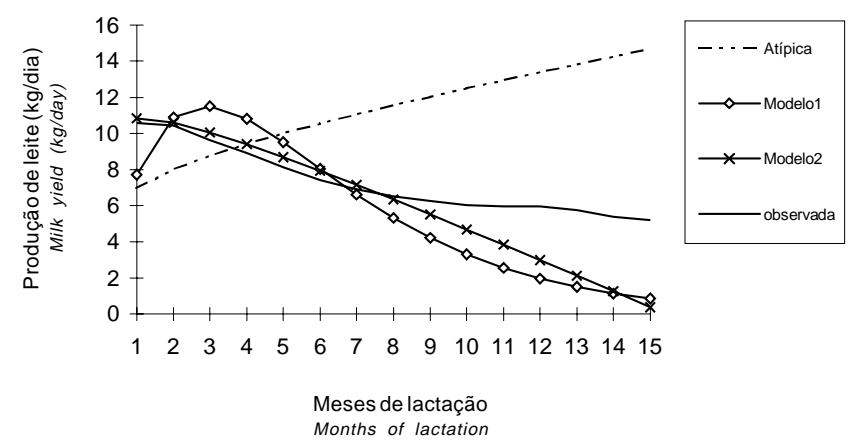

Figura 2 - Curvas de lactação observada e estimada pelos modelos.

Figure 2 - Lactation curves observed and estimated by the models. 
1208 Rev. bras. zootec.

Tabela 3 - Distribuição dos coeficientes de determinação $\left(R^{2}\right)$, segundo a ordem de parto

Table 3 - Distribution of the coefficients of determination $\left(R^{2}\right)$, according to parturity order

\begin{tabular}{lrrrrrrrrr}
\hline $\begin{array}{l}\text { Modelo } \\
\text { Model }\end{array}$ & \multicolumn{1}{c}{$\mathrm{R}^{2}$} & Op1 & Op2 & Op3 & $\begin{array}{c}\text { Op4 } \\
(\%)\end{array}$ & Op5 & Op6 & Op7 & Op8 \\
\hline & $R^{2}$ & & & & & & & \\
& $<0,70$ & 68,9 & 57,4 & 48,7 & 55,5 & 59,3 & 57,5 & 75,8 & 91,0 \\
1 & $0,70-0,79$ & 12,0 & 13,7 & 18,6 & 10,4 & 13,2 & 19,7 & 10,4 \\
& $0,80-0,89$ & 16,6 & 20,8 & 24,8 & 26,2 & 16,6 & 12,2 & 13,8 & 4,5 \\
& $>0,90$ & 2,5 & 8,1 & 7,9 & 7,9 & 10,9 & 10,6 & - \\
& $0,70-0,79$ & 13,0 & 17,1 & 11,4 & 12,7 & 21,9 & 16,7 & 25,0 & 28,7 \\
2 & $0,80-0,89$ & 28,5 & 22,7 & 29,0 & 20,6 & 18,8 & 21,2 & 21,4 & 4,7 \\
& $>0,90$ & 18,4 & 34,0 & 34,6 & 40,5 & 31,9 & 33,3 & 14,3 & 4,7 \\
\hline
\end{tabular}

Op $=$ ordem de parto

$O p=$ parturity order

Tabela 4 - Produção de leite total observada (PLTO), período de lactação (PLACT) e estimativas dos parâmetros "a" e "c" dos modelos, de acordo com a ordem de parto

Table 4 - Observed mean values for total milk yield (PLTO), lactation length (PLACT) and estimates for the parameters "a" and "c", according to parturity order

\begin{tabular}{|c|c|c|c|c|c|c|}
\hline $\begin{array}{l}\text { Ordem de parto } \\
\text { Parturity order }\end{array}$ & "a"1 & "c"1 & "a"2 & "c" $" 2$ & PLTO & PLACT \\
\hline 1 & 8,97 & 0,32 & 10,09 & 0,77 & 2318,31 & 306,33 \\
\hline 2 & 11,33 & 0,35 & 11,87 & 0,98 & 2427,93 & 292,04 \\
\hline 3 & 11,85 & 0,37 & 11,96 & 1,03 & 2350,55 & 283,13 \\
\hline 4 & 12,09 & 0,37 & 11,99 & 1,04 & 2283,19 & 275,42 \\
\hline 5 & 13,29 & 0,39 & 12,62 & 1,14 & 2333,31 & 269,36 \\
\hline 6 & 12,96 & 0,39 & 12,70 & 1,10 & 2469,31 & 283,41 \\
\hline 7 & 11,48 & 0,39 & 11,02 & 0,98 & 2068,37 & 265,44 \\
\hline 8 & 11,86 & 0,34 & 12,86 & 0,88 & 3008,17 & 299,72 \\
\hline
\end{tabular}

"a"1 , "c"1, "a"2 e "c"2 - parâmetros estimados pelos modelos 1 e 2, respectivamente.

"a"1, "c"1, "a"2 and "c"2 - parameters estimated by the models 1 and 2, respectively.

A relação entre produção inicial e taxa de declínio da produção pode ser observada no decorrer dos anos (Figuras 3 e 4). A variação desses parâmetros, ano a ano, indica que tais características são influenciadas principalmente por fatores de ambiente do que por fatores genéticos. Tais resultados podem ter sido em razão de diferenças na alimentação e no manejo, na temperatura e na precipitação pluviométrica, influenciando a quantidade e qualidade das forrageiras oferecidas aos animais ao longo dos anos. Dessa maneira, formatos mais desejáveis de curva de lactação poderiam ser conseguidos com a melhoria, por parte dos produtores, de fatores como nutrição e manejo.

Nas Tabelas 7 e 8 são apresentadas as correlações genéticas e ambientais entre as características produção total de leite e produção em 305 dias e os parâmetros dos modelos 1 e 2, respectivamente.

As correlações genéticas entre os parâmetros " $a$ " e " $c$ " foram positivas para os modelos 1 e 2 $(0,45$ e 0,75$)$. Este resultado sugere que filhas de touros com maior valor genético para produção inicial " $a$ " tendem a apresentar declínio mais acentuado na produção de leite ao longo da lactação “ $c$ ”. Dessa forma, a seleção para a produção inicial resultaria em vacas com maior taxa de declínio da produção. Este resultado foi semelhante ao relatado por SHANKS et al. (1981) e oposto ao encontrado por GRAVERT e BAPTIST (1976).

Ainda para as mesmas características, as correlações ambientais encontradas $(0,75$ e 0,70$)$ foram altas e positivas, indicando que as condições ambientais e os efeitos dos fatores não-aditivos foram semelhantes na sua influência sob ambas as características. Assim, a melhoria nas condições de manejo e alimentação poderia atenuar o antagonismo fenotípico entre produção inicial e taxa de declínio da produção de leite. Resultados opostos, no entanto, foram obtidos por VARONA et al. (1998).

Pode-se observar nas Tabelas 7 e 8 que as correlações genéticas da produção de leite total e o 


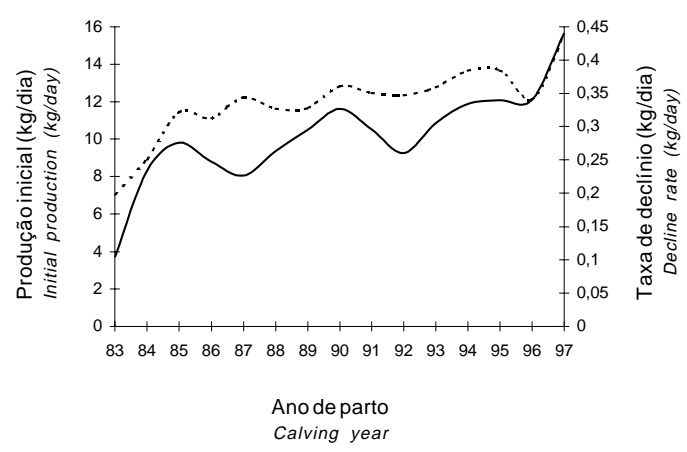

Figura 3 - Produção inicial de leite "a" e taxa de declínio da produção "c" estimadas pelo modelo 1, de acordo com o ano de parto.

Figure 3 - Initial milk production "a" and production decline rate "c" estimated by the model 1, according to calving year.

parâmetro " $c$ " apresentaram sentidos contrários nos modelos estudados.

BIANCHINI SOBRINHO e DUARTE (1988) ao analisarem registros de produção de leite de vacas Gir, obtiveram estimativas positivas para a correlação entre estes parâmetros. No entanto, VARONA et al. (1998), usando métodos bayesianos, encontraram correlações genéticas de $-0,04$ entre a produção de leite total e a taxa de declínio da produção.

As correlações ambientais entre a taxa de declínio da produção e a produção de leite total foram negativas. Estes resultados sugerem que as causas da variação genética e ambiental influenciam estas características por meio de diferentes mecanismos fisiológicos.

A produção inicial mostrou ser positivamente correlacionada com a produção de leite total. Resul-

Tabela 5 - Valores médios observados para a produção de leite total (PLTO) e estimativas do parâmetros " $c$ " dos modelos, segundo a classe de produção inicial estimada

Table 5 - Observed mean values for the total milk yield (PLTO) and estimates to the parameters " $c$ " of the models, according to estimated initial production class

\begin{tabular}{cccc}
\hline $\begin{array}{l}\text { Produção inicial } \\
\text { Initial production }\end{array}$ & "c"1 & "c"2 & PLTO \\
\hline$\leq 5,0$ & 0,25 & 0,33 & 1271,99 \\
$5,0-10,0$ & 0,32 & 0,65 & 1950,90 \\
$10,0-15,0$ & 0,36 & 0,98 & 2674,91 \\
$>15,0$ & 0,48 & 1,56 & 2887,67 \\
"c"1 e "c"2 - parâmetros estimados pelos modelos 1 e 2, \\
" respectivamente. \\
$c$ "1 and "c" - parameters estimated by the models 1 and 2, respectively.
\end{tabular}

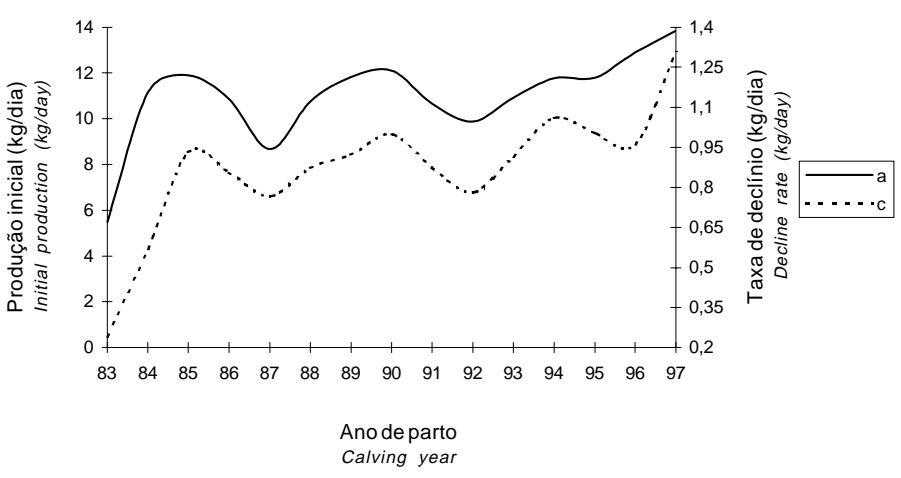

Figura 4 - Produção inicial de leite "a" e taxa de declínio da produção "c" estimadas pelo modelo 2 , de acordo com o ano de parto.

Figure 4 - Initial milk production "a" and production decline rate "c" estimated by the model 2 , according to calving year.

tado semelhante foi encontrado, no Brasil, por BIANCHINI SOBRINHO e DUARTE (1988), ao ajustarem o modelo linear hiperbólico aos dados de produção de leite de um rebanho da raça Gir.

De modo geral, pode-se inferir que a seleção para produção de leite provocaria acréscimo na produção inicial e pequenas mudanças no declínio da produção de leite das vacas.

As correlações genéticas e ambientais entre a produção em 305 dias (P305) e a taxa de declínio da produção, em direção e em magnitude, seguiram a mesma tendência das correlações obtidas para a produção de leite total. Entretanto, apresentaram magnitudes diferentes entre os modelos estudados.

Correlações genéticas e ambientais negativas entre produção de leite em 305 dias e o parâmetro " $c$ " também foram observadas por SCHNEEBERGER

Tabela 6 - Valores médios observados para produção de leite total (PLTO) e estimativas para os parâmetros "a" e "c", de acordo com o período de lactação (PLACT)

Table 6 - Observed mean values for total milk yield (PLTO) and estimates for the parameters "a" and "c", according to lactation length (PLACT)

\begin{tabular}{ccccccc}
\hline $\begin{array}{l}\text { PLACT } \\
\text { (Meses) }\end{array}$ & $\begin{array}{c}\text { PLTO } \\
(\mathrm{kg})\end{array}$ & \multicolumn{2}{c}{$\begin{array}{c}\text { Modelo 1 } \\
\text { Model 1 }\end{array}$} & & \multicolumn{2}{c}{$\begin{array}{c}\text { Modelo 2 } \\
\text { Model 2 }\end{array}$} \\
(Month) & & "a" & "c" & & "a" & "c" \\
\hline $5-7$ & 1418,76 & 13,79 & 0,48 & & 11,11 & 1,29 \\
$7-9$ & 1821,02 & 11,77 & 0,40 & & 10,91 & 1,06 \\
$9-11$ & 2478,40 & 10,50 & 0,33 & & 11,49 & 0,88 \\
$11-13$ & 3209,55 & 10,17 & 0,29 & & 12,23 & 0,82 \\
$>13$ & 3094,34 & 7,05 & 0,25 & & 9,81 & 0,59 \\
\hline
\end{tabular}


1210 Rev. bras. zootec.

Tabela 7 - Correlações genéticas (acima da diagonal) e correlações ambientais (abaixo da diagonal) da produção total de leite observada (PLTO), produção em 305 dias (P305) e parâmetros ("a" e " $c$ ") obtidos para o modelo 1

Table 7 - Genetic (above the diagonal) and environmental correlations (below the diagonal) of total milk yield (PLTO), 305-days yield (P305) and parameters "a" and "C" obtained for the model 1

\begin{tabular}{|c|c|c|c|c|}
\hline $\begin{array}{l}\text { Característica } \\
\text { Trait }\end{array}$ & " $a$ " & “c” & PLTO & P305 \\
\hline " $a "$ & - & 0,45 & 0,55 & 0,56 \\
\hline “c” & 0,75 & - & $-0,19$ & $-0,19$ \\
\hline PLTO & $-0,07$ & $-0,53$ & - & 0,98 \\
\hline P305 & $-0,01$ & $-0,48$ & 0,96 & - \\
\hline
\end{tabular}

(1981), nos Estados Unidos, em vacas da raça PardoSuíça e, no Brasil, por GADINI et al. (1998), em um rebanho comercial de vacas mestiças. Esses autores concluíram que o modelo gama incompleto forneceu parâmetros e características que podem servir como critério de seleção de maneira tão eficiente quanto a produção em até 305 dias, com a vantagem dessas características serem obtidas na primeira metade da lactação.

As correlações amostrais (Pearson) entre produção de leite total e a produção inicial foram de 0,67 e 0,73 , respectivamente, para cada modelo, indicando associação linear favorável entre os valores genéticos preditos. Entretanto, para a produção de leite total na lactação e a taxa de declínio da produção, essas correlações foram de $-0,13$ e 0,50 . As correlações de ordem (Spearman) obtidas entre as danificações dos animais quanto a produção de

Tabela 8 - Correlações genéticas (acima da diagonal) e correlações ambientais (abaixo da diagonal) da produção de leite total (PLTO), produção em 305 dias (P305) e parâmetros "a" e "c" obtidos para o modelo 2

Table 8 - Genetic (above the diagonal) and environmental correlations (below the diagonal) of total milk yield (PLTO), 305-days yield (P305) and parameters "a" and "c" obtained for the model 2

\begin{tabular}{lcccc}
\hline $\begin{array}{l}\text { Característica } \\
\text { Trait }\end{array}$ & " & “c” & PLTO & P305 \\
\hline “a” & - & 0,75 & 0,86 & 0,87 \\
“c” & 0,70 & - & 0,38 & 0,37 \\
PLTO & 0,47 & $-0,18$ & - & 0,98 \\
P305 & 0,56 & $-0,12$ & 0,96 & - \\
\hline
\end{tabular}

leite total e a produção inicial foram altas e positivas (0,68 e 0,74$)$, sugerindo que os indivíduos com os maiores valores genéticos para produção inicial seriam também aqueles com maiores valores para produção de leite total. As correlações de ordem entre a produção de leite total e a taxa de declínio foram de 0,03 e $-0,54$, respectivamente, para os modelos estudados.

\section{Conclusões}

De modo geral, pode-se inferir que a seleção para a produção de leite total na lactação causa acréscimo na produção inicial e mudanças inexpressivas na taxa de declínio da produção de leite, não alterando, assim, o formato da curva de lactação. No entanto, vacas da raça Guzerá com maiores produções iniciais apresentam declínios na produção de leite mais acentuados ao longo da lactação.

Possivelmente, alterações no formato da curva de lactação dos rebanhos estudados seriam obtidas com maior sucesso, por meio da melhoria nas condições de ambiente a que os animais são submetidos. A seleção direta para menores taxas de declínio da produção seria de baixa eficiência.

\section{Referências Bibliográficas}

BATRA, T.R., LIN, C.Y., McALLISTER, A.J. et al. 1987. Multitrait estimation of genetic parameters of lactation curves in Holstein heifers. J. Dairy Sci., 70(10):2104-2111.

BIANCHINI SOBRINHO, E., DUARTE, F.A.M. 1988. Genetic and environmental aspects of the linear hyperbolic lactation curve. Rev. Bras. Genet., 11(3):671-678.

BOLDMAN, K.G., KRIESE, L.A., VAN VLECK, L.D. 1995. A Manual for use of MTDFREML; a set programs to obtain estimates of variances and covariances [DRAFT]. Lincoln: Department of Agriculture, Agricultural Research Service. 115p.

CARDOSO,V.L., BENINTENDI, R.P., FREITAS, M.A.R. Estudo sobre a curva de lactação de um rebanho da raça Gir de seleção leiteira. Pico de produção e persistência da lactação. In: REUNIÃO ANUAL DA SOCIEDADE BRASILEIRA DE ZOOTECNIA, 23, 1986, Campo Grande, MS. Anais... Campo Grande: SBZ, 1986. p.281.

COBUCI, J.A., EUCLYDES, R.F., VERNEQUE, R.S. et al. 2000 Curva de lactação na raça Guzerá. Rev. bras. zootec. 29(5):1332-1339.

DURÃES, M.C., TEIXEIRA, N.M., FREITAS, A.F. 1991. Curvas de lactação de vacas da raça Holandesa mantidas em confinamento total. Arq. Bras. Med. Vet., 43(5):447-458.

EL FARO, L., ALBUQUERQUE, L.G., FRIES, L.A. 1999. Comparações de alguns modelos matemáticos para ajuste à curva de lactação média de um rebanho da raça Caracu. Rev. bras. zootec., 28(5):987-992.

FERRIS, T.A., MAO, I.L., ANDERSON, C. R. 1985. Selecting for lactation curve and milk yield in dairy cattle. J. Dairy Sci. 68(6):1438-1448. 
GADINI, C.H., EL FARO, L., FREITAS, M.A.R. Seleção para produção de leite auxiliada pela curva de lactação de vacas mestiças. In: REUNIÃO ANUAL DA SOCIEDADE BRASILEIRA DE ZOOTECNIA, 35, 1998, Botucatu, SP. Anais... Botucatu: SBZ, 1998. p.291.

GRAVERT, H.O., BAPTIST, R. 1976. Breeding for persistency of milk yield. Livest. Prod. Sci., 3(1):27-31.

GROSSMAN, M., KUCK, A.L., NORTON, H.W. 1986. Lactation curves of purebred and crossbred dairy cattle. J. Dairy Sci., 69(1):195-203.

JUNQUEIRA, L.V., NEIVA, R.S., VEIGA, R.D. et al. 1997. Estudo das curvas de lactação de vacas Holandesas de alguns rebanhos do Estado de Minas Gerais, por intermédio de uma função gama incompleta. R. Soc. Bras. Zootec., 26(6):1109-1118.

KELLOGG, D.W., URQUHART, N.S., ORTEGA, A.J. 1977. Estimating Holstein lactation curves with a gamma curve. J. Dairy Sci., 60(8):1308-1315.

LOPES, M.A., NEIVA, R.S., VALENTE, J. et al. 1996. Aplicação da função tipo gama incompleta no estudo da curva de lactação de vacas da raça Holandesa, variedade preta-branca, mantidas em sistema intensivo de produção. R. Soc. Bras. Zootec., 25(6):1086-1101.

PAPAJCSIK, I.A., BODERO, J. 1988. Modeling lactation curves of Friesian cow in a subtropical climate. Anim. Prod., 47:201-207.

QUEIROZ, S.A., ALBUQUERQUE, L.G., FREITAS, M.A.R. et al. 1991. Fatores genético e de meio que influenciam os componentes da curva de lactação de bovinos da raça Holandesa. Arq. Bras. Med. Vet. Zootec., 43(4):357-370.
SCHNEEBERGER, M. 1981. Inheritance of lactation curve in Swiss brown cattle. J. Dairy Sci., 64:475-483.

SHANKS, R.D., BERGER,P.J., FREEMAN, A.E. 1981. Genetic aspects of lactation curves. J. Dairy Sci., 64:1852-1860.

VARONA, L., MORENO, C., CORTES GARCIA, L.A. et al. 1998. Bayesian analysis of Wood's lactation curve for Spanish dairy cows. J. Dairy Sci., 60(5):1469-1478.

VERNEQUE, R.S. Procedimentos numéricos e estimação de componentes de covariância pelo método da máxima verossimilhança restrita, na análise multivariada, aplicados a modelos de melhoramento animal. Piracicaba, SP: ESALQ, 1994. 157p. Tese (Doutorado em Estatística) - Escola Superior de Agricultura "Luiz de Queiroz" / Universidade de São Paulo, 1994.

Recebido em: 05/12/00

Aceito em: 02/04/01 